\title{
Autonom und unfallfrei: Betrachtungen zur Rolle der Technischen Aufsicht im Kontext des autonomen Fahrens ${ }^{1}$
}

\author{
Annika Dix*, Jens R. Helmert*, Thomas Wagner** \& Sebastian Pannasch* \\ * Technische Universität Dresden, Fakultät Psychologie \\ ** DEKRA e.V. Dresden, DEKRA Technology Center Klettwitz \\ Korrespondenzadresse: \\ Dr. Annika Dix \\ Ingenieurpsychologie und angewandte Kognitionsforschung \\ Fakultät Psychologie \\ Technische Universität Dresden \\ Helmholtzstr. 10 \\ 01062 Dresden \\ annika.dix@tu-dresden.de
}

\section{Zusammenfassung}

Die Entwicklung des autonomen Fahrens erfährt aktuell große Unterstützung, nicht zuletzt durch einen entsprechenden Gesetzentwurf der Bundesregierung. Der vorliegende Beitrag diskutiert die mit den Entwicklungen zum autonomen Fahren verbundenen

Herausforderungen. Im Fokus stehen dabei die zu erwartenden Aufgaben an die Technische Aufsicht, den Menschen, der in schwierigen Situationen Entscheidungen und Freigaben vornehmen muss. Für diese Aufgaben werden Aspekte von Gefahrenwahrnehmung sowie Aufgabenkomplexität betrachtet und aus arbeitspsychologischer Perspektive eingeordnet. Diese stärker individuelle Betrachtung wird in den Zusammenhang gestellt mit zu erwartenden strukturellen Herausforderungen, wie beispielsweise Mischverkehr und technischer Zuverlässigkeit. Der Beitrag schließt mit sieben Empfehlungen in Bezug auf die Gestaltung autonomen Fahrens, deren Erfüllung für die zukünftige Verkehrssicherheit von zentraler Bedeutung erscheinen.

Schlüsselwörter: autonomes Fahren, technische Aufsicht, Gefahrenwahrnehmung, Situationsbewusstsein

\footnotetext{
1 Der vorliegende Text wurde gefördert durch die Deutsche Forschungsgemeinschaft (DFG) im Rahmen der Exzellenzstrategie des Bundes und der Länder - EXC 2050/1 - Projektnummer 390696704 - als Exzellenzcluster „Centre for Tactile Internet with Human-in-the-Loop“ (CeTI) der Technischen Universität Dresden.
} 


\begin{abstract}
The development of autonomous driving is currently strongly supported also by a corresponding draft law by the German government. This article discusses the challenges associated with the development of autonomous driving. The present paper focuses on the expected tasks for the remote operator. The remote operator is, according to the draft law, the human being who has to make decisions and approvals in difficult situations. For these tasks, aspects of hazard perception and task complexity are considered from the perspective of occupational psychology. This more individual consideration is placed in the context of expected structural challenges, such as mixed traffic and technical reliability. The article concludes with seven recommendations regarding the design of autonomous driving. The implementation of these recommendations seems to be of central importance for future road safety.
\end{abstract}

Keywords: autonomous driving, remote operator, hazard perception, situational awareness

\title{
1 Einleitung
}

Aktuell gibt es zahlreiche Diskussionen über die zukünftige Gestaltung von Mobilität. Dabei spielen neben der Sicherheit die Fragen der Bedarfe, der Technologien und der gesellschaftlichen Trends eine wesentliche Rolle (Weber, 2020). Im Mai 2021 hat die Bundesregierung den Gesetzentwurf „zur Änderung des Straßenverkehrsgesetzes und des Pflichtversicherungsgesetzes - Gesetz zum autonomen Fahren“ (BT-Drucks. 19/27439, 2021) vorgelegt, welcher den Einsatz fahrerloser Kraftfahrzeuge im öffentlichen Straßenverkehr - z. B. sogenannter People-Mover (automatisierte Personentransportsysteme) - ermöglicht. Der Gesetzentwurf wird ergänzt durch Ausführungsvorschriften, die sich derzeit noch im Status eines Referentenentwurfs im Bearbeitungsstand befinden, und angedachte Festlegungen zu Verfahrensregelungen über die Erteilung von Betriebserlaubnissen für Kraftfahrzeuge mit autonomer Fahrfunktion, über die Genehmigung von festgelegten Betriebsbereichen sowie zu Anforderungen und Sorgfaltsvorschriften für die am Betrieb von Kraftfahrzeugen mit autonomer Fahrfunktion beteiligten Personen enthalten (Referentenentwurf „Autonome Fahrzeug-Genehmigungsund Betriebsverordnung" AFGBV, 2021).

Der vorliegende Beitrag konzentriert sich auf die Darstellung und Bewertung verschiedenster Herausforderungen, die mit den aktuellen Bestrebungen im Kontext des autonomen Fahrens verbunden sind. Beginnen wir dazu mit einem Blick in die Zukunft, der uns zu folgendem Szenario führen könnte:

„Die Koffer sind gepackt, die Freude auf den Urlaub ist groß und die Fahrt zum Flughafen ist gebucht. Mit einem autonomen Fahrzeug kann sich die ganze Familie einfach und bequem zum gewünschten Zielort bringen lassen. Damit beginnt der Urlaub bereits jetzt. Da, eine Nachricht auf dem Handy, es kann losgehen."

So in etwa könnte eine Werbung für die Nutzung eines autonomen Fahrzeugs mit dem höchsten Automatisierungsgrad bei Fahrzeugen gestaltet sein. Dies wäre ein Fahrzeug, welches unter allen Fahr- und Umgebungsbedingungen vollständig automatisiert geführt ist. Im Fahrzeug selbst gibt es keinerlei Möglichkeiten zur Steuerung und Bedienung. Es 
handelt sich also um ein Auto ohne Lenkrad, bei dem ein Eingriff durch die Fahrgäste weder vorgesehen noch möglich ist. Gemäß der nationalen und internationalen Einteilung wäre dies ein Fahrzeug mit einer Automatisierung ab Stufe 4 (Society of Automotive Engineers SAE, 2021).

Wie ist es inzwischen der Familie aus dem obigen Szenario ergangen?

„Die Strecke zum Flughafen ist kurz und lässt sich bei guter Verkehrslage in ca. 20 min bewältigen. Aber ausgerechnet heute, bei der Abfahrt in den Urlaub, scheint es wie verhext. Erst gab es Stop \& Go auf einer der Ausfallstraßen aus der Stadt, und jetzt blockiert eine Tagesbaustelle die Weiterfahrt. Fahrzeuge können nur durch Überfahren der durchgezogenen Linie passieren. Bei autonomen Fahrzeugen kann so etwas nur die technische Aufsicht entscheiden."

Technische Aufsicht? Was verbirgt sich hinter diesem Begriff und wozu benötigt ein selbstfahrendes Auto eine Technische Aufsicht? Derartige Fahrzeuge, welche mit Hilfe zahlreicher Überwachungssensoren und künstlicher Intelligenz selbständig fahren, können in Situationen geraten, in denen Algorithmen keine selbständige Entscheidung treffen können. Genau für diese Situationen steht ein Mensch als Technische Aufsicht (TA) bereit: Benötigt ein selbstfahrendes Auto Beistand bei einer Entscheidung, beispielsweise wenn ein Ausweichmanöver ein kurzfristiges Übertreten einer Straßenverkehrsregel erfordert (z. B. Überfahren einer durchgezogenen Linie), wird die Freigabe eines solchen Fahrmanövers durch die TA erforderlich, die dadurch die strategische Kontrolle sensu Michon (1986) über das Fahrzeug übernimmt. Daneben verpflichtet die Novelle des Straßenverkehrsgesetzes die TA zur Deaktivierung des Kraftfahrzeugs mit autonomer Fahrfunktion, sofern das Fahrzeug nicht in der Lage ist, die Fahraufgabe selbstständig zu bewältigen (BT-Drucks. 19/27439, 2021, S. 9). Außerdem kann die TA alternative Fahrmanöver vorschlagen. Hinter dem Begriff der TA verbirgt sich also ein menschlicher Entscheider, der Zugriff auf die sensorischen Informationen des Fahrzeugs bekommen und in der Situation eine Entscheidung fällen kann, um die Schwierigkeiten aufzulösen und eine Weiterfahrt zu ermöglichen. Ein Eingriff der Passagiere ist unter keinen Umständen vorgesehen. So stellt es sich auch in unserem Szenario dar.

„Die Weiterfahrt verzögert sich um wenige Augenblicke, wir bitten um Ihr Verständnis erklingt eine Stimme aus dem Off. Neugierig schauen wir aus dem Fenster, um die Ursache für den überraschenden Zwischenstopp ausfindig zu machen."

In diesem Moment fragen sich nicht nur die Passagiere, was wohl die Fahrt behindert. Auch die TA muss sich einen Überblick über die Situation verschaffen. An dieser Stelle ergeben sich gleich mehrere Problemfelder für die Einführung autonomer Fahrfunktionen auf Deutschlands Straßen.

Aus kognitionspsychologischer Perspektive lässt sich das Führen eines Fahrzeuges als Aufgabenbearbeitung auf verschiedenen hierarchischen Stufen darstellen (vgl. Rasmussen 1990; Michon, 1986). Obwohl die Anforderungen durch die Fahraufgabe mit steigender Fahrerfahrung sinken, spielen regel- und wissensbasierte Prozesse stets eine wesentliche Rolle. Die Gefahrenvermeidung und Vorbeugung unfallursächlichen Fehlverhaltens setzt somit eine nicht unbedeutende kognitive und psychomotorische Kompetenz des Fahrers 
voraus. So ist es auch nicht verwunderlich, dass 93,5 \% der Unfallursachen auf menschliches Fehlverhalten zurückzuführen sind, wie die GIDAS-Datenbank (German InDepth Accident Study, https://www.gidas.org) ausweist. In der GIDAS-Datenbank werden, auf Initiative der Bundesanstalt für Straßenwesen (BASt) und der Forschungsvereinigung Automobiltechnik (FAT), seit 1973 Verkehrsunfälle gesammelt und ausgewertet.

Ein möglicher Lösungsansatz, das Problem menschlichen Fehlverhaltens zu reduzieren, besteht in der Automatisierung einzelner Fahraufgaben bzw. in der autonomen Fahrzeugführung. Aus psychologischer Perspektive bedeutet diese Automatisierung, speziell für den Aufgabenbereich der TA, allerdings eine grundlegende Veränderung unseres bisherigen Verständnisses von Verantwortung und Kontrolle im Straßenverkehr. So wichtig Innovation und Fortschritt im Bereich des Straßenverkehrs auch sein mögen, zum aktuellen Zeitpunkt ist kaum abzusehen, ob die Einführung von selbstfahrenden Fahrzeugen wirklich zur vielfach versprochenen Senkung der Unfallrate führen wird. Hierfür müssen für autonome Fahrzeuge folgende Voraussetzungen zur Gefahrenabwehr erfüllt sein (vgl. Haupt, 2021): (i) sie weisen keine erhöhte Fehleranfälligkeit im Vergleich zu konventionellen Fahrzeugen auf, (ii) sie reagieren ebenso zuverlässig auf widrige Straßenverhältnisse wie menschliche Fahrer, und (iii) sie kommunizieren mit schwächeren Verkehrsteilnehmern (z. B. Fußgänger, Kinder, Radfahrer). Die Erfüllung dieser Voraussetzungen wird zumindest in der Rechtswissenschaft eher zurückhaltend beurteilt (Haupt, 2021). Darüber hinaus bestehen große Bedenken, wie der Arbeitsplatz einer TA gestaltet wird.

Der vorliegende Artikel setzt sich daher mit folgenden Punkten auseinander: In Kapitel (2) Tätigkeitsfeld der TA stehen die Herausforderungen zukünftiger TAs im Mittelpunkt. Dabei betrachten wir neben allgemeinen arbeitspsychologischen Perspektiven auch die spezifische Verlagerung des Kontroll- und Überwachungsdilemmas vom Fahrer zur TA. Kapitel (3) Strukturelle Herausforderungen beschäftigt sich mit der prinzipiellen Einbettung autonomen Fahrens in den Kontext von Mobilität. Dazu zählen neben der Organisation von Mischverkehr auch Fragen von Verantwortung, Schuld und Haftung. Im abschließenden Kapitel (4) Diskussion und Ausblick werden die zentralen Gedanken noch einmal integriert.

\section{Tätigkeitsfeld der TA}

Die TA befindet sich nicht im Fahrzeug, sondern sehr wahrscheinlich in einer büroähnlichen Umgebung, z. B. einer Leitstelle, die vom Halter des Fahrzeugs bereitgestellt wird (vgl. AFGBV, 2021, S. 107 f.), und reagiert auf Betriebsstörungen, die das Fahrzeug meldet. Vermutlich wird eine TA meist mit einfachen Teilproblemen konfrontiert, die sie zunächst identifiziert, bevor entschieden und gehandelt wird. Ist beispielsweise die Fahrspur versperrt, kann die TA die Freigabe zum Überfahren einer durchgezogenen Linie erteilen, damit das Hindernis passiert werden kann. Meldet die Abstandsmessung bei Regen häufige Fehlalarme für Hindernisse, kann die Empfindlichkeit der Sensorik angepasst werden. Fraglich ist allerdings auf Basis welcher Informationen die TA entscheidet und woher sie weiß, dass ein Hindernis sicher über die Gegenspur passiert werden kann oder die Sensorik Fehlalarme produziert und kein Stau vorliegt, der regelmäßiges Abbremsen verlangt. 
Bislang ist das Tätigkeitsfeld der TA nur unzureichend beschrieben, auch der aktuelle Gesetzentwurf der Bundesregierung liefert dazu keine konkreten Aussagen. Die nachfolgende Beschreibung klingt eher nach Science-Fiction, repräsentiert aber ein Szenario mit optimalen Voraussetzungen, welches durch eine TA möglicherweise gelöst werden könnte. Wir wollen deshalb für dieses Szenario Faktoren identifizieren, die den Erfolg einer TA maßgeblich mitbestimmen, aber in der aktuellen Gesetzesvorlage bisher keine explizite Berücksichtigung erfahren.

„Auf dem Bildschirm über dem Kontrollpanel im Aufsichtsraum des Control Centers for Automated Road Transport (CART) erscheint ein Warnhinweis: FZ-372E Fahrspur versperrt - Fahrt unterbrochen. Darunter steht: Achtung, durchgehende Fahrbahnbegrenzung. Zusätzlich zeigt ein rot leuchtender Kontrollschalter eine Handlungsaufforderung an. Die TA bestätigt mit dem Schalter die Übernahme, drückt eine weitere Taste auf dem Kontrollpanel und mehrere Kameraansichten auf die Umgebung von Fahrzeug FZ-372E erscheinen auf dem Bildschirm. Die Frontkamera liefert Bilder von einigen vorausfahrenden Fahrzeugen und einer Baustellenabsperrung. Es zeigt sich, dass die vorausfahrenden Fahrzeuge über die durchgezogene Begrenzungslinie auf die Gegenspur fahren, um die Baustelle zu umfahren. Die Darstellung der Heckkamera lässt erkennen, dass sich hinter dem Fahrzeug FZ-372E eine Warteschlange gebildet hat, deren Ende die TA nicht sehen kann. Es gibt keinen Hinweis darauf, wann die Baustellendurchfahrt für den Gegenverkehr frei gegeben wird. Da der Abstand zum vorausfahrenden Fahrzeug nicht allzu groß scheint, entscheidet sich die TA im Auswahlmenü links für die Option Spurenwechsel trotz Fahrbahnbegrenzung und gibt durch einen weiteren Tastendruck die Fahrt wieder frei."

Im beschriebenen Szenario liefern Kamerabilder der TA die Informationen zum Verkehrsgeschehen. Auf dieser Basis muss sie die Situation beurteilen und eine Entscheidung treffen. Im geschilderten Beispiel ist das Fahrzeug zuvor zum Stehen gekommen, befindet sich also im gesicherten Stillstand. Außerdem wird der TA, so wie im Gesetzentwurf vorgesehen, mindestens ein Fahrmanöver vorgeschlagen. Dies bedeutet, dass basierend auf den vom System über die Situation bereitgestellten Informationen der TA ein anforderungsgerechtes Handeln ermöglicht werden muss. In den nachfolgenden Kapiteln stellen wir die kognitionspsychologischen Grundlagen für ein solches Handeln dar, speziell im Kontext von verzögertem Situationsbewusstsein (2.1) und variierender Aufgabenkomplexität (2.2). Abschließend folgt eine arbeitspsychologische Bewertung des Tätigkeitsfeldes der TA (2.3).

\subsection{Gefahrenwahrnehmung und Situationsbewusstsein beim automatisierten Fahren}

Die Wahrnehmung von Gefahren erfordert die Erkennung der relevanten Informationen einer Situation, ein Verständnis über die vorliegenden Schwierigkeiten und die Ableitung möglicher Handlungsoptionen. Gemäß dieser Betrachtung ist Situationsbewusstsein (SB) die Grundlage für Gefahrenwahrnehmung. Die Definition nach Endsley (1995) unterscheidet drei Stufen von SB: (1) Wahrnehmen (2) Verstehen und (3) Projizieren. Entsprechend muss eine TA beispielsweise die Position und Dynamik eines Hindernisses sowie die des überwachten Fahrzeugs und anderer Fahrzeuge zunächst wahrnehmen können. Aus der Bedeutsamkeit einzelner Elemente im Gesamtkontext resultiert das 
Verstehen der Situation, etwa der versperrten Fahrspur, die trotz durchgezogener Linie verlassen werden muss. Daran anschließend erfolgt das Projizieren zukünftiger Handlungen (z. B. die Identifikation auftauchenden Gegenverkehrs als Gefahrenquelle) und die Vorhersage der Konsequenzen. Eine aktive Aufgabenbearbeitung bildet die Voraussetzung für ein angemessenes SB; gleichzeitig ist ausreichendes SB eine notwendige Bedingung für anforderungsgerechtes Entscheiden und angemessenes Handeln. Eine Beeinträchtigung des SB kann durch die Entkopplung von der Aufgabe entstehen, beispielsweise durch die Automatisierung von (Teil-)Aufgaben. Anders ausgedrückt kann von den Passagieren im autonomen Fahrzeug keine SB erwartet werden, da sie - ähnlich wie Passagiere in einem Taxi - dem Geschehen im Straßenverkehr vermutlich nur wenig Aufmerksamkeit widmen.

Eine derartige Entkopplung von der Aufgabe kann zum Out-of-the-Loop (aus der Schleife, nicht mehr in aktuelle Prozesse involviert) Phänomen führen (vgl. Merat et al., 2019). Während ein steuernder Fahrer im Fahrzeug fortlaufend Informationen zum Verkehrsgeschehen bekommt und verarbeitet, wird eine TA sehr plötzlich mit einem Problem konfrontiert. Dies erfolgt in der Funktion eines passiven Beobachters mit selektivem Informationsangebot, welches sich quantitativ, qualitativ und in der zeitlichdynamischen Entwicklung stark von dem eines aktiven Kraftfahrers unterscheidet. Die TA wird sich vermutlich an relativ abstrakten Parametern orientieren und muss auf fehlende Informationen und Ereignisse schließen. Dies macht die Informationsverarbeitung der TA fehleranfällig, was der Gesetzgeber offensichtlich bereits einkalkuliert hat, da für die TA eine Haftpflichtversicherung verlangt wird, wobei die Haftungsgrenzen bei autonomen Fahrzeugen im Fall von Personenschäden bei zehn Millionen Euro liegen sollen und bei Sachschäden mit zwei Millionen Euro veranschlagt werden (Haupt, 2021). Diese Haftungsgrenzen entsprechen dem Doppelten der Grenzen bei konventionellen Fahrzeugen.

Fehleinschätzungen der TA können z. B. die Fahrgeschwindigkeit betreffen. Die Beurteilung der Geschwindigkeit anderer Fahrzeuge aus dem Gegenverkehr durch Kraftfahrzeugführer "in-the-loop" schwankt selbst unter experimentellen Bedingungen ganz erheblich und variiert zwischen 50 \% Unterschätzung und $13 \%$ Überschätzung (Meyer-Gramcko, 1980). Je nachdem, ob der Beobachter eine Entfernungsschätzung aus der Fahrgastzelle eines Pkw oder daneben auf einem Stuhl (vergleichbar der Situation einer TA in einer Leitstelle) vornimmt, differieren die Schätzungen um bis zu 29\%, selbst wenn alle sonstigen experimentellen Randbedingungen konstant gehalten werden (Moeller et al., 2016). Eine derartig fehlerhafte Nutzung von Informationen, d. h. Orientierungsfehler oder Fehleinschätzungen (z. B. des Abstands oder der Geschwindigkeit), sind jedoch unfallkausale Ursachen, wie Staubach (2009) bei einer Tiefenanalyse von 474 Unfällen feststellte. Die Autorin übertrug das Fehlerklassifikationsmodell der menschlichen Informationsverarbeitung von Hacker (1998) auf den Kontext Autofahren und fand zudem, dass Fehler, die aufgrund eines objektiven Mangels an Informationen entstehen, z. B. in Form von Sichtbehinderungen durch Gebäude, Fahrzeuge oder durch die Witterung, ebenfalls die Unfallgefahr erhöhen (Staubach, 2009).

Diese Beispiele verdeutlichen, dass Verständnis und Interpretation objektiver Bedingungen einer Fahraufgabe ganz wesentlich von der aktuellen Wahrnehmung, dem Feedback während der Verhaltensausführung sowie von Erfahrungen und Erwartungen des Fahrers 
abhängen. Diese Einflussfaktoren bilden die Grundlage für das Mensch-Maschine-UmweltSystemverständnis, wobei inkorrekte, undifferenzierte und lückenhafte Repräsentationen die Handlungsregulation beeinträchtigen; darauf hingewiesen haben bereits Müller et al. (2018) im Zusammenhang mit automatisierter Fahrfunktion auf Stufe 3. Im Vergleich zu automatisiertem Fahren der Stufe 3 verschärft sich die Illusion der Wahrnehmungsbereitschaft beim autonomen Fahren mit einer TA aufgrund der räumlichen Entkoppelung von der eigentlichen Fahraufgabe. Umso wichtiger ist die Identifikation der möglichen Problemstellungen, mit denen eine TA konfrontiert werden wird, sowie eine Prüfung auf deren Lösbarkeit. Die Aufgaben einer TA sollten entsprechend vor der Einführung autonomer Fahrzeuge im Straßenverkehr definiert werden.

Studien zu SB bei Übernahmesituationen durch Fahrer im automatisierten Fahrzeug zeigen insgesamt eine deutliche Verzögerung (Mok et al., 2017). Während SB auf Stufe 1 (wahrnehmen) noch vergleichsweise schnell aufgebaut werden kann (5-8 s), liegt die Dauer auf Stufe 2, gerade wenn es darum geht das Verhalten anderer Verkehrsteilnehmer zu verstehen, bereits bei über 20 s (Lu et al., 2017). Diese Zahlen sind beachtenswert. Bis die TA in der obigen Beispielsituation das Fahrgeschehen in der Gegenspur verstanden hat und das Alternativmanöver freigeben kann, hat sich aus der ohnehin schon angespannten Stop \& Go Situation der Anfang eines Staus entwickelt. Durch jedes weitere autonome Fahrzeug, das nachfolgt und bei dem die Freigabe durch eine TA erteilt werden muss, würde sich diese Situation weiter verschärfen und vermutlich bei konventionellen Fahrzeugführern einen gefährlichen „Überholdruck“ fördern.

Tatsächlich wurde in Studien für einen Remote Operator, also einem Fahrzeugführer außerhalb des Fahrzeuges analog zur TA, in Abhängigkeit vom Problem sogar ein verzögertes SB von $29 \mathrm{~s}$ bis über $162 \mathrm{~s}$ festgestellt (Scholtz et al., 2004). Zum Vergleich: In $162 \mathrm{~s}$ legt ein Auto, das mit einer Geschwindigkeit von $30 \mathrm{~km} / \mathrm{h}$ fährt, eine Strecke von $1,35 \mathrm{~km}$ zurück, bei $120 \mathrm{~km} / \mathrm{h}$ sogar 5,4 km und im Stand auf einer zweispurigen Autobahn lassen sich in dieser Zeit weit über 200 vorbeifahrende Autos registrieren. Diese Tatsachen illustrieren die Dynamik von Verkehrssituationen, welche die TA bei ihrer Entscheidung berücksichtigen muss. Entsprechend sieht der Gesetzentwurf auch vor, dass die technische Ausrüstung entsprechende Handlungsaufforderungen mit "ausreichender Zeitreserve“ „optisch, akustisch oder sonst wahrnehmbar" anzeigen kann (BT-Drucks. 19/27439, 2021, S. 8). Auf welcher Basis die technische Ausrüstung zu einer entsprechenden Einschätzung kommt, wird nicht spezifiziert. Insofern scheint der Umgang des Gesetzgebers mit aktuell nicht prüfbaren technischen Anforderungen fraglich: „Um die Triebkraft des automatisierten, autonomen und vernetzten Fahrens nicht zu verlangsamen, soll, soweit gewisse technische Anforderungen noch nicht überprüfbar sind, diesbezüglich für eine Übergangszeit die Herstellererklärung nach § 1f Absatz 3 genügen." (BT-Drucks. 19/27439, 2021, S. 24). Durch Beilegen dieser zum Betriebshandbuch wird „den Nutzerinnen und Nutzern entsprechender Fahrzeuge somit ein vertrauensstiftender Nachweis zur Sicherheit dieser neuen Mobilitätsform gegeben“. Für die Herstellererklärung sieht das Bundesministerium für Verkehr und digitale Infrastruktur gemäß der AFGBV vor, dass in einem Sicherheitskonzept „relevante gefährliche Szenarien und Ereignisse identifiziert und in einer Risikoanalyse bewertet werden" (AFGBV, 2021, S. 46). Die Vollständigkeit des Szenarienkatalogs muss der Hersteller durch entsprechende Validierungsfahrten belegen. 
Es bleibt also auch für Hersteller zu hoffen, dass die Komplexität des Straßenverkehrs tatsächlich in diesem Katalog möglichst umfangreich abgebildet werden kann.

Neben der Problematik des verzögerten SB und dessen Auswirkungen auf die Handlungsfähigkeit einer TA, bleibt ebenfalls unklar, inwiefern durch den Gesetzgeber eine gleichzeitige Überwachung und/oder Unterstützung mehrerer Fahrzeuge durch eine TA erlaubt bzw. vorgesehen ist. Die Zuordnung mehrerer Fahrzeuge zu einer TA ist aus Unternehmerperspektive naheliegend, zumal die TA nicht zur ständigen Überwachung eines im autonomen Betrieb befindlichen Fahrzeuges verpflichtet sein soll (BT-Drucks. 19/27439, 2021, S. 8). Hierbei gilt zu berücksichtigen, dass im Fall einer Beeinträchtigung des autonomen Fahrzeugs und Handlungsaufforderung an die TA der Aufbau von SB neben Zeit auch kognitive Ressourcen erfordert. Dies kann zur Reduktion der Arbeitsleistung für andere Aufgaben führen. So investierten beispielsweise in einer Usability-Studie zu Suchund Rettungsrobotern die Remote Operators bei der Robotersteuerung $30 \%$ ihrer Zeit ausschließlich in das Erlangen und Aufrechterhalten von SB (Yanco \& Drury, 2004). Folglich sind Regelungen notwendig, wie die (nicht ständige) Überwachung weiterer Fahrzeuge im Fall einer Übernahmesituation erfolgen soll. Eine potenzielle Überforderung der TA wird im Allgemeinen vor allem von der Aufgabenart bestimmt sein. Im Folgenden werden denkbare Aufgaben einer TA und die Rolle der Aufgabenkomplexität näher betrachtet.

\subsection{Aufgabenkomplexität und Verantwortungsbereiche im Tåtigkeitsfeld der TA}

Der Gesetzentwurf zum autonomen Fahren lässt offen, welche Verantwortlichkeiten einer TA zugewiesen werden, also welche Aufgaben konkret übernommen werden sollen und damit auch mit welcher Aufgabenkomplexität eine TA konfrontiert sein wird. Mögliche Vorkommnisse wie das Überfahren von Ampeln auf Dauer-Rot werden im Gesetzentwurf dargestellt. Zudem ist geregelt, dass Fahrzeuge ihre Systemgrenzen kennen und in der Lage sind, sich „selbständig in einen risikominimalen Zustand zu versetzen, wenn die Fortsetzung der Fahrt nur durch eine Verletzung des Straßenverkehrsrechts möglich wäre“ (BT-Drucks. 19/27439, 2021, S. 8). Das sind die Momente, in denen die TA ins Spiel kommt. „Der Begriff „Systemgrenzen“ ist nicht näher definiert“ und „bewusst technikoffen gehalten“ (BT-Drucks. 19/27439, 2021, S. 23). Die Grenze der Leistungsfähigkeit eines Fahrzeuges kann allerdings je nach Hersteller variieren. Mit dieser Offenheit bestätigt der Entwurf ein weiteres Mal die Ironien der Automatisierung (Bainbridge, 1983): das Machbare wird automatisiert, übrig bleiben Aufgabenteile mit zu hoher Komplexität, die fortan von der TA erfüllt werden müssen. Das grundlegende Problem hinter der Vision von weniger Verkehrsunfällen durch autonomes Fahren bleibt somit bestehen und wird ad absurdum geführt. Die Ursachen für Unfälle verschieben sich vom menschlichen Versagen des Fahrers im Auto zum menschlichen Versagen des Designers. Dieses Versagen soll durch den Einsatz einer weiteren Person, der TA, ausgeglichen werden, die wiederum neuen Fehlerquellen ausgesetzt ist (Merat \& Louw, 2020).

Aufgrund der zu erwartenden Bandbreite an Technologien des autonomen Fahrens mit entsprechend variierenden „Systemgrenzen“, sowie der Komplexität des Straßenverkehrs, ist von einer hohen Heterogenität hinsichtlich der Anforderungen an eine TA auszugehen. Es stellt sich somit die Frage, wer in welcher Situation welche Verantwortlichkeit 
übernehmen kann und sollte (vgl. auch Graewe, 2021). In Abhängigkeit von der jeweils an eine TA übergebenen Verantwortlichkeit können sich verschiedene Anforderungen an die vorauszusetzenden Fähigkeiten ergeben. Beispielsweise ist das Erkennen und Umfahren von Hindernissen eine Tätigkeit, die in der Regel nur auf dem Automatisierungslevel Null in diesem Fall vom Fahrer im Auto - übernommen werden muss und einen Führerschein voraussetzt. Kann diese Tätigkeit auch an eine TA übergeben werden? Diese soll laut AFGBV über eine entsprechende Fahrerlaubnis der jeweiligen Fahrzeugklasse verfügen (AFGBV, 2021, S. 55). Also alles kein Problem oder sollte die TA weitere Voraussetzungen erfüllen? Und für welche Situationen könnten diese Überlegungen relevant werden?

Nehmen wir an ${ }_{\llcorner}$die Tagesbaustelle im genannten Szenario führt streckenweise nicht über die Gegenfahrbahn, sondern über einen Fahrstreifen, der sich genau zwischen der Fahrzeug- und der Gegenspur befindet, es muss also auf der durchgezogenen Begrenzungslinie gefahren werden. Diese Situation wirkt zunächst nicht gefährlicher als die Situation, die unsere TA aufgelöst hat. Allerdings muss das Fahrzeug nun über einen längeren Zeitraum gegen straßenverkehrsrechtliche Regeln verstoßen. Es ist anzunehmen, dass nicht jede technische Ausrüstung diese Aufgabe erfüllen kann. In diesem Fall schließt das „die Zulässigkeit der Nutzung autonomer Fahrfunktionen nicht aus“, da diese „auch dann zulässig ist, wenn sie innerhalb des festgelegten Betriebsbereichs die an die Fahrzeugführung gerichteten Verkehrsvorschriften erfüllen können“ (BT-Drucks. 19/27439, 2021, S. 21). Sollte sich das autonome Fahrzeug also in einer solch unerwarteten Situation selbst deaktivieren? Oder hätte es gar nicht eingesetzt werden dürfen? Wie können geeignete Betriebsbereiche identifiziert werden, für die keine unerwarteten Situationen auftreten? Ist das Fahrzeug erst einmal in die Baustellendurchfahrt eingefahren, ist Stehenbleiben zumindest keine geeignete Lösung. Allgemeiner formuliert sind zum jetzigen und letztlich zu keinem Zeitpunkt alle Situationen vorher beschreibbar es wird immer eine Restmenge an unerwarteten Situationen geben.

In den USA ist es möglich einem Remote Operator die Fernsteuerung eines Fahrzeuges (im Sinne einer Teleoperation) zu erlauben (Mutzenich et al., 2021). Sollte also die TA das Fahrzeug durch besagten Streckenabschnitt der Baustelle steuern können? Dies ist im deutschen Gesetzentwurf derzeit noch nicht vorgesehen und würde tatsächlich weit höhere Anforderungen an die Ausbildung der TA und die technischen Lösungen stellen. Derzeit scheint beabsichtigt, dass bei Erreichen der Betriebsgrenzen das Fahrzeug in den risikominimalen Zustand versetzt und die Fahrt durch manuelle Steuerung durch einen Fahrer im Fahrzeug oder außerhalb im Nahfeld des Fahrzeuges (maximale Distanz: $6 \mathrm{~m}$ ) fortgesetzt wird (AFGBV, 2021, S. 32 \& 51). Für den Halter, beispielsweise Betreiber im ÖPNV, resultiert daraus, dass zunächst ein höherer Personalkostenaufwand zu kalkulieren ist. Probleme einer Teleoperation über größere Distanzen wären vor allem die eingeschränkten Informationen und die Latenz zwischen Handlung und Rückmeldungen für die TA. Durch das reduzierte SB verlängert sich bereits für Übernahmesituationen im Fahrzeug die Reaktionsgeschwindigkeit von 1 auf über 3 s (Eriksson \& Stanton, 2017). Bei einer Fahrzeuggeschwindigkeit von $30 \mathrm{~km} / \mathrm{h}$ bedeutet das im Fall von plötzlich auftretenden Hindernissen einen ungebremsten Fahrweg von $25 \mathrm{~m}$ bis zur tatsächlichen Initiierung der Bremsung. Eine Übernahme muss entsprechend vorbereitet werden und selbst dann ergeben sich für die TA außerhalb des Fahrzeugs im Aufsichtsraum des Kontrollzentrums noch zusätzliche Herausforderungen. 
Systemverzögerungen bei der Übermittlung der Sensordaten zur TA und der Durchführung von Steuerungsbefehlen durch das Fahrzeug können das Kontrollempfinden und die Steuerungsleistung massiv beeinträchtigen (Neumeier et al., 2019). Im Luftverkehr werden für zeitkritische Szenarien, die eine präzise Steuerung des Flugzeuges erfordern, Verzögerungen von insgesamt maximal $100 \mathrm{~ms}$ als annehmbar betrachtet (Bailey et al., 2004). Bei über 240 ms kann die Kontrolle des Flugzeuges nicht mehr gewährleistet werden. Eine derart schnelle Informationsübertragung - gerade bei der zu erwartenden Komplexität an erforderlichen sensorischen Daten - setzt eine entsprechende und störungsfreie Infrastruktur voraus, beispielsweise schnelle und sichere Netzwerke auch in ländlichen Gebieten. Um die TA mit allen für die sichere Fernsteuerung relevanten Informationen in einem zeitlich angemessenen Rahmen versorgen zu können, ist zudem ein umfangreiches Wissen über die Prinzipien der menschlichen Wahrnehmung und des zielgerichteten Verhaltens in der Mensch-Maschine Interaktion - also einem Kontext, der hinsichtlich der üblicherweise in unserer Umwelt vorliegenden Informationen abweicht erforderlich. Hier besteht derzeit noch großer Forschungsbedarf.

Inwieweit beispielsweise die bisher verfügbaren technischen Lösungen den dynamischen Prozess der menschlichen Gefahrenwahrnehmung und -vermeidung auf allen Entfernungsstufen, Fahrersichtachsen und damit verknüpften Fixationsverläufen adäquat simulieren können, ist bislang nicht belegt. Pradhan und Crundall (2016) integrierten neuere Erkenntnisse zur experimentellen Gefahrenwahrnehmungsforschung in das SBModell von Endsley (1995) und schlagen verschiedene, dynamisch aufeinanderfolgende Verarbeitungsstufen in Abhängigkeit von der Entfernung und den Raumwahrnehmungsbereichen des Fahrers vor. In einer Aufmerksamkeitszone („vigilance zone"), z.B. mehrere hundert Meter vor dem Fahrzeug, tauchen erwartungsinkongruente Vorboten einer Gefahr am Fluchtpunkt auf. Diese sind noch zu weit weg, um Gefahrenhinweise extrahieren zu können, erhöhen jedoch die Aufmerksamkeit des Fahrers und werden für nachfolgende Prüfungen „vorgemerkt". Im näheren Orientierungsbereich („strategic zone") werden Hinweise auf Gefahrenreize fixiert, gewichtet und deren Verlauf antizipiert. Möglicherweise erfolgt eine erste Gegenreaktion, z.B. Verringerung der Geschwindigkeit oder ein Fahrstreifenwechsel als Schutzmaßnahme gegen die potentielle Gefährdung. Auf der nächsten Wahrnehmungsstufe („tactical zone“) wird der Gefahrenreiz umfassend verarbeitet und es erfolgt eine Reaktionsauswahl. In dem am nächsten liegende Raumwahrnehmungsbereich („operational zone“) wird nur noch reagiert und der Unfall vermieden oder es kommt zur Kollision. Wenn der TA nur ausgewählte Ausschnitte dieser zusammenhängenden und dynamischen Verkehrssituation auf unterschiedlichen Bildschirmen präsentiert werden, besteht das Risiko, dass sicherheitsrelevante Hinweise zu spät oder gar nicht erkannt werden.

Die in vielen Aspekten reduzierten Informationen, die einer TA bereitgestellt werden können, bergen zudem das Risiko eines fehlenden Embodiments (UNECE, 2020), d. h. die TA wird die Bedeutung ihrer Handlungen ähnlich wie in einem Computerspiel nicht fühlen können (Mutzenich et al., 2021). Dies kann mit einem reduzierten Verantwortungsgefühl einhergehen, vor allem aber zu Missverständnissen durch Fehleinschätzung der Bedeutung einzelner Informationen führen. Beispielsweise wird Bewegungswahrnehmung durch die Limitierung auf durch Kameras erfasste visuelle Informationen kombiniert mit abstrakten Parametern wie Geschwindigkeitsangaben bedeutsam erschwert. Auch kann eine fehlende 
Rückmeldung über akustische Informationen aus der Umwelt - ähnlich wie bei zu lauter Musik für einen Fahrer im Fahrzeug - zum Übersehen relevanter Informationen führen (vgl. z. B. Dalton \& Behm, 2007)

Einige Probleme, die sich durch die abweichende Wahrnehmung der TA ergeben, lassen sich vermutlich durch die Unterstützung mittels automatisierter Fahrfunktionen, also eine Teilautomatisierung, beheben. So kann eine Kollisionsvermeidung bei eingeschränkter Wahrnehmung auch technisch umgesetzt werden, allerdings sind dafür entsprechende Richtlinien erforderlich. Zudem antizipieren wir für die Durchführung bestimmter Manöver speziell ausgebildete Fachkräfte wie im Flugverkehr, d.h. eine Art Spezialeinsatzgruppe für Notfälle bzw. Hochrisikosituationen. Es gibt verschiedene Ansätze der TA den Umgang mit solchen Situationen zu erleichtern bzw. überhaupt zu ermöglichen. Wir wollen unser Szenario fortsetzen und ein fiktives Notfallmanagement einführen, welches die Notwendigkeit für eine genaue Anforderungsanalyse des Tätigkeitsfeldes der TA und für die entsprechende Festsetzung vorauszusetzender Qualifikationen noch einmal veranschaulichen soll. Nach dieser Darstellung erfolgt eine zusammenfassende Bewertung des Tätigkeitsfeldes der TA.

\subsection{Arbeitspsychologische Bewertung des Tåtigkeitsfeldes der TA}

Schauen wir uns also das Ende unseres Szenarios für die TA bzw. die Mitarbeiter des Notfallmanagements an, wobei anzumerken ist, dass die Art der Arbeitsteilung zwischen beiden Funktionen bislang durch den Gesetzentwurf nicht berührt wird (BT-Drucks. 19/27439, 2021).

„Der Mitarbeiter des Notfallmanagements hatte sich gerade im Stuhl zurückgelehnt, als der Alarm eingeht. Der Bildschirmhintergrund verschwindet und der Mitarbeiter befindet sich inmitten einer dicht befahrenen Baustellendurchfahrt auf dem Zubringer zu einem Flughafengelände. Das Fahrzeug, das sich gerade deaktiviert hat, scheint zu stehen mit leichter Orientierung Richtung rechter Baustellenbegrenzung. Der Virtual-Reality Bildschirm zeigt die Perspektive des Fahrers aus dem Fahrzeug. Im unteren Bereich des Sichtfeldes ist im Vordergrund ein Warnhinweis zu sehen: FZ-372E Weiterfahrt nicht möglich. Darunter leuchtet in roten Buchstaben: Remote Control aktivieren. Da keine weiteren Warnhinweise vorliegen, kann der Mitarbeiter die manuelle Steuerung bestätigen. Zunächst werden jedoch die Fahrgäste über den Steuerungsvorgang informiert, mit dem Hinweis sich ruhig zu verhalten. Der Mitarbeiter ist mit der TA im Aufsichtsraum per Voice-Chat verbunden und kann neben den Kamera- und Toninformationen aus dem Fahrzeug auch direkt Kontakt mit den Passagieren aufnehmen. Umgekehrt haben die Passagiere auch die Möglichkeit jederzeit einen Notruf an die Mitarbeiter des Notfallmanagements zu senden. Der Mitarbeiter hatte die Baustelle wiedererkannt und auch direkt die durchgezogene Fahrbahnbegrenzung in der Mitte der Baustellenspur entdeckt. Langsam und sicher steuert er, von seinem Arbeitsplatz im Großraumbüro mit seinem Lenkrad in den Händen und dem Head-Mounted Display auf dem Kopf, wie bereits mehrere Male an diesem Vormittag auch das Fahrzeug FZ-372E durch den kritischen Streckenabschnitt."

Technische Lösungen im Bereich der virtuellen oder erweiterten Realität können helfen, der TA einerseits ein möglichst vollständiges Abbild der Verkehrssituation zu vermitteln; auf diese Weise wird es für die TA leichter, sich in die Situation hineinzuversetzen bzw. 
wird es möglich zusätzliche Informationen zu geben, die den Umgang mit den Herausforderungen erleichtern. Anforderungen an diese Technologien, um der TA zur erfolgreichen Erfüllung ihrer Aufgaben dienen zu können, sind basierend auf der bisherigen Studienlage nicht in ausreichendem Umfang formulierbar. Die Entwicklung in diesem Feld zeigt noch verschiedenste Probleme auf, beispielsweise nachteilige Einflüsse von Latenz (Bailey et al., 2004). Die Herausforderungen an technische Lösungen sind hierbei aufgrund der erhöhten Menge an zu übertragenden Informationen besonders groß. Ein weiteres Problem, das u.a. auch im Zusammenhang mit Latenzeinflüssen steht, aber auch generell mit Widersprüchen in der Sinneswahrnehmung (z. B. visueller Bewegungseindruck ohne tatsächliche Bewegung), ist die Motion oder auch Cybersickness. Insgesamt sollten bei der Diskussion dieser Lösungsansätze entsprechend auch immer Konsequenzen für das Anforderungsprofil der TA abgeleitet werden. Dieses sowie weitere arbeitspsychologische Aspekte des Tätigkeitsfeldes der TA sollten vor Einführung autonomer Fahrzeuge auf deutschen Straßen genau betrachtet, beschrieben und möglichst auch optimiert werden.

Ein wichtiges Argument für Automatisierung in allen Branchen ist neben der Sicherheit die Entlastung der Arbeitenden, heutzutage vor allem im Hinblick auf mentale Belastung. Mentale Unterforderung kann aber langfristig ebenso zu gesundheitlichen Problemen führen wie Überlastung (Shultz et al., 2010). Hancock und Warm (1989) beschreiben optimale Belastung als eine umgekehrte U-Funktion, mit der größten physiologischen und psychologischen Anpassungsfähigkeit in der Mitte, und dem größten Stressempfinden an den Rändern. Diese Idee wurde von Young und Stanton (2002) in der malleable theory of attention (Formbare Theorie der Aufmerksamkeit) aufgegriffen und erweitert, indem sie aufzeigen, dass geringere Belastung auch mit geringeren Kapazitäten zur Bewältigung von Anforderungen einhergehen. Das bedeutet, dass eine aktivierte Person eine zusätzliche Anforderung leichter bewältigt als eine unterforderte Person. Solche Kapazitätseinbußen durch Unterforderung können zu Überforderungsszenarien führen (Byrne, 2011), wenn plötzlich Leistung abgefordert wird. Es besteht die Gefahr, dass die Arbeitssituation einer TA genau diese Merkmale aufweisen wird: Unterforderung in ruhigen Zeiten verbunden mit Spitzen, in denen mehrere Fahrzeuge gleichzeitig abgefertigt und Probleme mit unterschiedlicher Priorisierung gelöst werden müssen.

Zudem ist anzunehmen, dass die Tätigkeiten von TAs sowohl tagsüber als auch nachts in Anspruch genommen werden, insbesondere mit zunehmender Anzahl autonom fahrender Fahrzeuge. Daraus resultiert die Anforderung von Schichtarbeit, die eine Belastung des zirkadianen Rhythmus darstellt (Reinberg \& Ashkenazi, 2008) und zu Müdigkeit sowie verminderter Leistungsfähigkeit führen kann (Books et al., 2017; Flynn-Evans et al., 2016). Die Beeinträchtigungen der Leistungsfähigkeit äußern sich beispielsweise in der Zunahme von Fehlern (de Cordova et al., 2016) aber auch verminderter psychomotorischer Wachsamkeit (Behrens et al., 2019). Insbesondere diese beiden Leistungskriterien sind für den Tätigkeitsbereich der TA von grundlegender Bedeutung für die zuverlässige Aufgabenbearbeitung.

Wie sich wohl die Fahrgäste in der beschriebenen Situation gefühlt haben?

„Nachdem sich der Signalton abgestellt hatte und der Mitarbeiter uns das weitere Vorgehen erklärt hat, bewegen wir uns langsam vorwärts und verlassen bald darauf die Baustellendurchfahrt. Der Mitarbeiter bedankt sich für die Unterstützung und wünscht uns 
eine gute Weiterfahrt. Wir haben den Schreck bald überwunden und freuen uns auf den anstehenden Urlaub. Nur ein kleines Gefühl der Unsicherheit bleibt: Würde es ein Fahrer nicht vielleicht doch leichter haben."

\section{Strukturelle Herausforderungen}

Bislang wurden sicherheitskritische Effekte des autonomen Fahrens nur auf der Ebene von individuellen TAs während der Tätigkeitsausübung diskutiert. Diese Effekte werden durch Kontextbedingungen entsprechend verstärkt, dazu zählen der zu erwartende Mischbetrieb mit Fahrzeugen auf unterschiedlichen Automatisierungsstufen (0-3), die Interaktion dieser verschiedenen Fahrzeuge, das Verhalten weiterer Verkehrsbeteiligter sowie Systemausfälle.

Egal wie schnell sich autonomes Fahren in der Verkehrsgemeinschaft durchsetzen wird, ein Mischbetrieb aus manuell betriebenen und automatisiert fahrenden Fahrzeugen ist für die kommenden Jahrzehnte anzunehmen. Eine im Auftrag des ADAC e.V. durchgeführte Studie prognostiziert erste nennenswerte Zulassungszahlen bei Neufahrzeugen, die autonom von Tür-zu-Tür fahren, erst ab 2040; für 2050 werden 0,5 bis 2,1 Mio. Fahrzeuge dieser Bauart angenommen (PrognosAG, 2018). Ob und in welchem Umfang autonome Fahrzeuge dann tatsächlich genutzt werden, ist heute nur schwer vorherzusagen. Die Studienlage ist komplex und vor allem bezüglich der erhobenen Zielvariablen wie Nutzerintentionen, Akzeptanz, Kaufbereitschaft, persönliche Meinung, Bewertung usw. sehr heterogen. Die Literaturanalyse von Becker und Axhausen (2017), die 16 Studien zwischen 2013 und 2016 umfasste, erlaubt nur vorsichtige und wenig exakte Schlussfolgerungen. Demnach zeigen 18 bis 68\% der Befragten eine positive Affinität zu autonomen Fahrzeugen. Diese ist bei jüngeren Probanden und solchen männlichen Geschlechts stärker ausgeprägt, ebenso bei Personen mit höherem Bedürfnis nach Nervenkitzel, Abwechslung und Abenteuer.

Die Verkehrsmittelwahl wird von rationalen Faktoren beeinflusst, z. B. frühere Erfahrungen mit dem dominanten Verkehrsmittel, den daraus resultierenden Nutzungserfahrungen sowie von Gewohnheitsbildung (Havlíčková \& Zámečník, 2020), aber auch von emotionalen Motiven. Das Selbstfahren als aktive Tätigkeit bietet einen intrinsischen Belohnungswert durch handlungsbegleitende Emotionen wie Freude oder Fahrspaß, z. B. für OldtimerBegeisterte, und wird zudem mit Vorstellungen über gesundheitliche Vitalität, Unabhängigkeit und Teilhabe am gesellschaftlichen Leben verknüpft. Diese identitätsstiftende Funktion wird besonders deutlich, wenn Kraftfahrzeugführenden der Führerschein entzogen wird; das resultierende Belastungserleben übersteigt sogar jenes für Trennung oder Arbeitslosigkeit (Kieschke et al., 2010). All dies dürfte die Euphorie für autonomes Fahren erheblich reduzieren, sodass die tatsächliche Nutzung von autonom fahrenden Fahrzeugen noch länger hinter dem Marktangebot zurückbleiben dürfte. Das Bundesministerium für Verkehr und digitale Infrastruktur rechnet in den kommenden fünf Jahren jährlich mit einer Zulassung von 320 Fahrzeugen mit autonomer Fahrfunktion deutschlandweit, wobei sich die Nachfrage vermutlich auf den ÖPNV-Bereich beschränken wird. (AFGBV, 2021, S. 73). Zusätzlich hemmen dürften auch die Gebühren von geschätzten 82.000 EUR zum Betrieb eines autonomen Fahrzeugs (Haupt, 2021). Für gewerbliche Halter im ÖPNV wird sogar ein Erfüllungsaufwand zur Befolgung der rechtlichen 
Vorschriften des Bundes von jährlich über 10 Millionen EUR erwartet (AFGBV, 2021, S. 79 ff.) - unter der Schätzung, dass es zumindest vier Fahrzeuge bedarf, um überhaupt einen entsprechenden Service an 365 Tagen im Jahr anbieten zu können. Insofern scheint die Annahme eines langjährigen Mischbetriebs eine sehr wahrscheinliche Perspektive.

Fachleute sehen in diesem Mischbetrieb eine Störung der „Harmonie des Verkehrsflusses“ mit weniger ausbalancierten Geschwindigkeits- und Abstandsprofilen als gegenwärtig (vgl. Gruber \& Sammer, 2019). Autonome Fahrzeuge werden mit deutlich niedrigerer Geschwindigkeit und größeren Abständen zum Vorderfahrzeug im Vergleich zu manuell betriebenen Fahrzeugen unterwegs sein, da sie per se alle Regeln der StVO umsetzen müssen. Diese „StVO-Verpflichtung“ wiederum eröffnet Verhaltensangebote für manuelle Fahrer, z. B. zum Überholen oder zum Einfahren in eine Lücke zwischen zwei Fahrzeugen. Laut Gesetzentwurf dürfen autonom betriebene Fahrzeuge nur in festgelegten Betriebsbereichen fahren, allerdings fehlen vielerorts verfügbare Kapazitäten für zusätzliche Fahrspuren, so dass ein Großteil der bestehenden Infrastruktur gemeinsam genutzt werden wird. Zudem ist die Frage nach möglichen Investitionen in infrastrukturelle Anpassung bisher offengehalten. Vorerst wird vom Bundesministerium für Verkehr und digitale Infrastruktur betont, „dass ein Kraftfahrzeug mit autonomer Fahrfunktion grundsätzlich in der Lage sein soll, auf der vorhandenen Infrastruktur betrieben werden zu können“ (AFGBV, 2021, S. 103). Die gemeinsame Nutzung im Mischbetrieb könnte zu weiteren Irritationen führen, denn manuelle Fahrer sind längst nicht so regeltreu wie vorgeschrieben. Überhöhte Geschwindigkeit, Vorfahrtsverletzungen, ungenügender Mindestabstand und unangepasstes Fahrverhalten gehören zu den typischen Regelverletzungen auf Deutschlands Straßen (ausführlich bei Wagner et al., 2018) und dürften häufige und vermutlich störende Eingriffe der Steuerungsautomatik im autonomen Fahrzeug erfordern. Ein geminderter subjektiver Fahrkomfort wäre mindestens die Folge, ggf. auch Verkehrskonflikte mit Schadenspotenzial, zumindest bei Berücksichtigung der ITSystemgrenzen sowie der Fehleranfälligkeit der Überwachungs- und Steuerungsautomatik.

Die gegenwärtigen Kompetenzen der IT-Systeme im Erkennen und der Verhaltensprognose von besonders verletzlichen und schützenswerten Menschen, darunter Kinder, Sehbehinderte und offensichtlich Körperbehinderte bzw. von Fußgängern oder Radfahrern allgemein, stecken noch in den Anfängen. Dadurch sind Konflikte zwischen manuellem und automatisiertem Fahrbetrieb abzusehen. Überdies setzt die Umsetzung der StVO das einwandfreie Funktionieren der Sensortechnik und der programmierten Entscheidungslogik voraus. Diese Funktionsweise ist jedoch fehleranfällig, Gründe hierfür bestehen in Hardwaremängeln (Fehler in Bauteilen, Verschleiß, Manipulation, Beschädigung), in der Erkennung situativer Kontextbedingungen (z. B. Tages- oder Wanderbaustellen), der zuverlässigen Überwachung von Umgebungsbedingungen trotz erschwerter Wahrnehmbarkeit (infolge Witterungsbedingungen, z. B. Schnee, Nebel, Regen) bzw. in schadhafter Infrastruktur (Schlaglöcher oder unterbrochene Fahrbahnmarkierungen). Bereits heute sind die Prävalenzraten für Systemausfälle infolge von Störungen der Sensorik oder der Hardware durchaus markant. Boggs und Kollegen (2020) analysierten die Berichte von 36 Unternehmen, die hochautomatisierte Fahrzeuge in Kalifornien testen und berichteten 2,72 Systemausfälle pro 100 gefahrener Kilometer im hochautomatisierten Modus. Die im Fahrzeug anwesenden Sicherheitsfahrer hatten die Defizite in der Systemsteuerung - darunter nicht-situationsangepasste Fahrmanöver, 
Probleme bei der Identifikation von Lichtzeichenanlagen oder verzögerte Informationsverarbeitung durch die Sensoren - rechtzeitig erkannt und die Fahrzeugführung übernommen. Mutzenich und Kollegen (2021) weisen darauf hin, dass bei autonom fahrenden Fahrzeugen alle 288 Meilen ein sicherheitskritischer Wahrnehmungsfehler der Sensorik auftrete. Auch mit diesen Herausforderungen wird sich die TA beschäftigen müssen, ggf. indem das Fahrzeug deaktiviert wird.

Als Zwischenfazit lässt sich feststellen, dass eine Vielzahl an meldepflichtigen

Betriebsstörungen den Alltag der TA sowohl in quantitativer als auch qualitativer Hinsicht prägen werden. Infolge des Mischbetriebs könnte das Sicherheitsniveau anhaltend Einbußen erleiden. Zudem müssen zusätzliche Erschwernisse einkalkuliert werden, z.B. wenn Betriebsstörungen aufgrund eines Systemfehlers nicht gemeldet werden oder „falsche Alarme“ die mentalen Ressourcen der TA unnötig binden, z. B., weil ein Passagier ohne Anlass den „Notruf" auslöst. Solche derzeit noch nicht antizipierbaren Ereignisse dürften die Aufgabenkomplexität und -dichte der TA weiter steigern.

\section{Diskussion und Ausblick}

Aus der Perspektive mitreisender Personen gleicht autonomes Fahren weitgehend der traditionellen Fahrgastbeförderung mit Taxi, Bus oder Mietlimousine. Allerdings wird beim autonomen Fahren auf einen in der Fahrgastzelle anwesenden Fahrer verzichtet. Dennoch muss in beiden Fällen eine sichere Beförderung der Fahrgäste gewährleistet sein, sodass sich ein Vergleich gesetzlicher Regelungen zur Gewährleistung dieser übergeordneten Zielvorgabe lohnt. Sofern ein gewerblicher Chauffeur die Ersterteilung oder Verlängerung einer Fahrerlaubnis zur Fahrgastbeförderung anstrebt, müssen besondere Anforderungen hinsichtlich gesundheitlicher Konstitution, psychofunktionaler Leistungsfähigkeit und persönlicher Zuverlässigkeit erfüllt sein. Hier sind die Anforderungen deutlich höher als beim Pkw-Fahrer, der seine Fahrerlaubnis lediglich für private Zwecke nutzt. In Umsetzung staatlicher Schutzpflichten gemäß Artikel 2 des Grundgesetzes darf der Fahrgast auf eine sichere und adäquate Transportleistung vertrauen (Hentschel et al., 2017). Dies soll gewährleistet werden, indem geplant ist, dass die TA über eine Führerscheinklasse verfügen soll, die der von ihr betreuten Fahrzeugart entspricht (AFGBV, 2021, S. 109). Dennoch kann das Konzept der TA - wie in den vorigen Kapiteln skizziert -derzeit noch nicht als hinreichend definiert und „verkehrstauglich“ angesehen werden. Damit Gefährdungen für mitreisende Personen in autonomen Fahrzeugen weitestgehend minimiert werden können, stellen wir nachfolgend relevante Fragen zur Gestaltung autonomen Fahrens bzw. unterbreiten Gestaltungsempfehlungen, damit die Verkehrssicherheit auch zukünftig ausreichend gewährleistet ist.

1. Das Informationsangebot für die TA muss so gestaltet sein, dass eine umfassende, reliable und valide Beurteilung der Entscheidungssituation möglich ist, damit zeitnah ein adäquates mentales Modell aktiviert bzw. entwickelt werden kann. Dazu könnte die Bildschirmdarstellung der Verkehrssituation durch markante Hinweise mittels eines „Head-Up-Displays“ ergänzt werden. Zudem sollte eine „Umkreisbeschränkung“ der TAZuständigkeit ebenso erwogen werden wie eine Ortskundeprüfung für diesen Zuständigkeitsbereich, z.B. $30 \mathrm{~km}$ um den Einsatzort der TA. Da die Fernsteuerung des 
Fahrzeugs dem Operateur in der Leitzentrale die größten Probleme bereiten dürfte, sollte die Fahrstrecke im „Fernsteuerungsmodus“ begrenzt werden bei vorgeschrieben niedriger Fahrgeschwindigkeit, sofern künftig eine Erweiterung des Aufgaben- und Verantwortungsbereichs der TA erwogen würde (vgl. AFGBV, 2021, S. 32). Eine derartige Ausweitung des Handlungsfeldes der TA lässt sich langfristig vermutlich nicht verhindern.

2. Das Tätigkeitsprofil der TA sollte einer fachlich fundierten arbeitspsychologischen Anforderungsanalyse unterzogen werden, woraus sich nachvollziehbar anforderungsbezogene Qualifikationen sowie Trainings- und Unterstützungsmaßnahmen ableiten lassen. Das Tätigkeitsfeld der TA lässt sich mit dem eines Fluglotsen vergleichen. wobei erschwerend vor allem drei Faktoren wirken dürften: (i) kürzere Distanzen zu anderen Verkehrsteilnehmern, (ii) geringere Zeitintervalle zum Entscheiden und Handeln und (iii) das Fehlen eines menschlichen Kommunikations- und Kooperationspartner vor Ort. Erst wenn entsprechende Ergebnisse zur Anforderungsanalyse vorliegen, können seriös und substantiiert formale Qualifikationsvoraussetzungen abgeleitet werden. Der Vorstoß in der Ausführungsverordnung hinsichtlich einschlägiger Abschlüsse (z.B. als Diplom-Ingenieur oder staatlich geprüfter Techniker) erscheint derzeit noch verfrüht.

3. Der Arbeitsplatz einer TA sollte ein menschengerechtes Arbeiten ermöglichen. Dafür müssen die vier Humankriterien „Ausführbarkeit“, „Schädigungslosigkeit“,

„Beeinträchtigungsfreiheit" und „Persönlichkeitsförderlichkeit" gegeben sein (vgl. Hacker, 1998). Die ersten drei Humankriterien dienen der Gesundheitsprävention und damit dem Erhalt der Leistungsfähigkeit, das vierte der persönlichen Entwicklung. Die Arbeitsorganisation sollte durch vollständige, durchschaubare, sinnhafte und gesundheitsförderliche Tätigkeitsinhalte mit hohem Handlungsspielraum geprägt sein (Richter, 2015), sodass dysfunktionale und pathogene neo-tayloristische Folgeeffekte weitestgehend vermieden werden. Diese dürften kausal sicherheitsrelevant sein. Gleichzeitig wirken diese Tätigkeitsinhalte motivierend, denn sie sagen $25 \%$ der subjektiven Arbeitsleistung sowie $45 \%$ des Arbeitsengagements voraus, wie Richter (2015) unter Verweis auf meta-analytische Studienergebnisse berichtet. Zudem sollte eine Gefährdungsbeurteilung im Kontext des betrieblichen Arbeits- und Gesundheitsschutzes (vgl. § 5 ArbSchG) durchgeführt werden.

4. Die Anforderungen an den Betriebsbereich für autonome Fahrzeuge müssen eindeutig geregelt werden. Bislang scheint hier noch vieles ungeklärt. Handelt es sich um rein räumlich oder zusätzlich durch bestimmte Kontextbedingungen ausgestaltete Straßenräume? Sollen vorhandene Straßenverkehrsanlagen im Mischbetrieb genutzt werden oder sind speziell konzipierte Gestaltungslösungen für autonomes Fahren angestrebt? Wie lässt sich gewährleisten, dass unbefugte Fahrzeuge oder Verkehrsteilnehmer nicht zum Betriebssicherheitsrisiko werden? Welche Infrastrukturmaßnahmen (physisch und digital) sind im Straßenbau erforderlich?

5. Klare und verständliche Kommunikation zwischen Fahrzeugen und Verkehrsteilnehmern bildet die Voraussetzung für flüssige und gefahrlose Verkehrsteilnahme, z. B. indem ein automatisiertes Fahrzeug für andere Verkehrsteilnehmer sichtbar anzeigt, dass es deaktiviert wurde, sich in der Interaktion mit der TA befindet oder per Fernsteuerung bewegt wird. 
6. Alle Aspekte des Datenschutzes sind besonders wichtig, insbesondere in Bezug auf Software-Updates und Cybersicherheit. Dies gilt vor dem Hintergrund, dass pro 1000 Programmierzeilen mindestens ein Fehler auftritt und somit eine hohe Anzahl periodisch ausgerollter Software-Updates zu erwarten ist. Dies wird deutlich an den diesbezüglichen Aktivitäten des Automobilproduzenten TESLA, wonach im Jahr 2020 ca. alle 2-3 Tage neue Updates „via air“ installiert wurden (La Manna et al., 2021). Der Datenschutz betrifft darüber hinaus auch die Interessen der Fahrgäste - welche Informationen sollen die Anbieter eines autonomen Fahrservices über ihre Kunden erhalten?

7. Die Überwachung und Kontrolle aller an der Umsetzung der Fahraufgabe beteiligten hard- und softwaretechnischen Systeme stellt neue Herausforderungen gemäß des „thirdParty-Prinzips“. Hier sind die einschlägigen Prüforganisationen mit ihrem Sachverstand gefordert. In die Überwachungszyklen sollten alle Software-Updates einbezogen werden.

Aus den genannten Punkten leiten sich eine Reihe normativer Regelungslücken ab. Die Wissenschaft um den Bereich der Mensch-Maschine-Schnittstelle sieht sich mit einer Vielzahl bislang ungeklärter Fragen konfrontiert, so dass mit einem erhöhten Forschungsbedarf gerechnet werden muss. Der im Februar eingeleitete Gesetzgebungsprozess nahm im Mai 2021 innerhalb einer Woche die parlamentarischen Hürden in Bundestag und Bundesrat, obwohl tangierte Fach- und Interessenskreise sowie Verbände die Gesetzesinitiative als stark verbesserungswürdig kritisiert hatten. Daher darf die praktische Umsetzung dieser neuen Vorschriften mit Spannung erwartet werden und es bleibt zu hoffen, dass politischer Ehrgeiz, technische Systemgrenzen und ökonomisches Profitstreben nicht zu Lasten des "Faktors Mensch" und einer ansteigenden Unfallstatistik gehen.

\section{Literatur}

AFGBV (2021). Referentenentwurf des Bundesministeriums für Verkehr und digitale Infrastruktur. Entwurf einer Verordnung zur Durchführung des Gesetzes zur Änderung des Straßenverkehrsgesetzes und des Pflichtversicherungsgesetzes. https://ec.europa.eu/growth/toolsdatabases/tris/index.cfm/de/search/?trisaction=search.detail\&year=2021\&num=3 44\&dLang=DE

Zugegriffen: 23. Juli 2021.

Bailey, R., Arthur Iii, J., \& Williams, S. (2004). Latency requirements for head-worn display S/EVS applications. Proceedings of SPIE - The International Society for Optical Engineering, 5424. https://doi.org/10.1117/12.554462

Bainbridge, L. (1983). Ironies of Automation. Automatica, 19(6), 775-779. https://doi.org/Doi 10.1016/0005-1098(83)90046-8

Becker, F., \& Axhausen, K. W. (2017). Literature review on surveys investigating the acceptance of automated vehicles. Transportation, 44(6), 1293-1306. https://doi.org/10.1007/s11116-017-9808-9

Behrens, T., Burek, K., Pallapies, D., Kösters, L., Lehnert, M., Beine, A., Wichert, K., Kantermann, T., Vetter, C., Brüning, T., \& Rabstein, S. (2019). Decreased psychomotor 
vigilance of female shift workers after working night shifts. PLoS One, 14(7), e0219087. https://doi.org/10.1371/journal.pone.0219087

Boggs, A. M., Arvin, R., \& Khattak, A. J. (2020). Exploring the who, what, when, where, and why of automated vehicle disengagements. Accident Analysis \& Prevention, 136, 105406. https://doi.org/https://doi.org/10.1016/j.aap.2019.105406

Books, C., Coody, L. C., Kauffman, R., \& Abraham, S. (2017). Night shift work and its health effects on nurses. The Health Care Manager, 36(4), 347-353. https://doi.org/10.1097/hcm.0000000000000177

BT-Drucks. 19/27439 (2021). Entwurf eines Gesetzes zur Änderung des Straßenverkehrsgesetzes und des Pflichtversicherungsgesetzes - Gesetz zum autonomen Fahren. https://dserver.bundestag.de/btd/19/274/1927439.pdf Zugegriffen: 23. Juli 2021.

Byrne, A. (2011). Measurement of mental workload in clinical medicine: A review study. Anesthesiology and Pain Medicine, 1(2), 90-94. https://doi.org/10.5812/kowsar.22287523.2045

Dalton, B. H., \& Behm, D. G. (2007). Effects of noise and music on human and task performance: A systematic review. Occupational Ergonomics, 7, 143-152. https://doi.org/10.3233/OER-2007-7301

de Cordova, P. B., Bradford, M. A., \& Stone, P. W. (2016). Increased errors and decreased performance at night: A systematic review of the evidence concerning shift work and quality. Work, 53(4), 825-834. https://doi.org/10.3233/wor-162250

Endsley, M. R. (1995). Toward a theory of situation awareness in dynamic systems. Human Factors, 37(1), 32-64.

Eriksson, A., \& Stanton, N. A. (2017). Takeover time in highly automated vehicles: Noncritical transitions to and from manual control. Human Factors, 59(4), 689-705. https://doi.org/10.1177/0018720816685832

Flynn-Evans, E., Gregory, K., Arsintescu, L., \& Whitmire, A. (2016). Risk of performance decrements and adverse health outcomes resulting from sleep loss, circadian desynchronization, and work overload: Evidence Report. Natl. Aeronaut. Space Admin.

Graewe, D. (2021). Autonomes Fahren-Traum der Ingenieure, Alptraum der Juristen? 26. Interdisziplinäre Wissenschaftliche Konferenz Mittweida, Mittweida.

Gruber, C. J., \& Sammer, G. (2019). Erwartungen, verkehrspolitische Auswirkungen und Handlungsbedarf für automatisierte Fahrzeuge und Mobilitätsdienste. Straßenverkehrstechnik Themenheft Automatisierte Mobilität, 245-254.

Hacker, W. (1998). Fehlhandlungen und Handlungsfehler. In W. Hacker (Ed.), Allgemeine Arbeitspsychologie. Psychische Regulation von Arbeitstätigkeiten. Huber.

Hancock, P. A., \& Warm, J. S. (1989). A dynamic model of stress and sustained attention. Hum Factors, 31(5), 519-537. https://doi.org/10.1177/001872088903100503

Haupt, T. (2021). Auf dem Weg zum autonomen Fahren - der Entwurf eines Gesetzes zum Autonomen Fahren. Neue Zeitschrift für Verkehrsrecht (NZV) 04/2021, 172-177.

Havlíčková, D., \& Zámečník, P. (2020). Considering habit in research on travel mode choice: A literature review with a two-level methodology. Transactions on Transport Sciences, 11(1), 18-32. https://doi.org/10.5507/tots.2020.004

Hentschel, P., König, P., \& Dauer, P. (2017). Straßenverkehrsrecht (44 ed.). C. H. Beck. Kieschke, U., Kieschke, T., \& Schubert, W. (2010). Fahrerlaubnisentzug als kritisches Lebensereignis. Zeitschrift für Verkehrssicherheit 03/2010, 143-148. 
La Manna, M., Treccozzi, L., Perazzo, P., Saponara, S., \& Dini, G. (2021). Performance evaluation of attribute-based encryption in automotive embedded platform for secure software over-the-air update. Sensors, 21(2), 515. https://www.mdpi.com/1424-8220/21/2/515

Lu, Z., Coster, X., \& de Winter, J. (2017). How much time do drivers need to obtain situation awareness? A laboratory-based study of automated driving. Applied Ergonomics, 60, 293-304. https://doi.org/https://doi.org/10.1016/j.apergo.2016.12.003

Merat, N., \& Louw, T. (2020). Allocation of function to humans and automation and transfer of control. In Handbook of Human Factors for Automated, Connected, and Intelligent Vehicles. (pp. 153-173). CRC Press.

Meyer-Gramcko, F. (1980). Die Schätzung der Fahrgeschwindigkeit. Der Sachverständige, 4(1), 2-12.

Michon, J. A. I. E. (1986). A critical view of driver behavior models: What do we know, what should we do? In L. Evans \& R. C. Schwing (Eds.), Human Behaviour and Traffic Safety. (pp. 485-524) Springer US.

Moeller, B., Zoppke, H., \& Frings, C. (2016). What a car does to your perception: Distance evaluations differ from within and outside of a car. Psychonomic Bulletin \& Review, 23(3), 781-788. https://doi.org/10.3758/s13423-015-0954-9

Mok, B., Johns, M., Miller, D., \& Ju, W. (2017). Tunneled in: Drivers with active secondary tasks need more time to transition from automation. In Proceedings of the $2017 \mathrm{CHI}$ Conference on Human Factors in Computing Systems (pp. 2840-2844). Association for Computing Machinery. https://doi.org/10.1145/3025453.3025713

Müller, K., Reimann, C., \& Wagner, T. (2018). Automatisiertes Fahren - Neue Anforderungen an die Kraftfahreignung? Zeitschrift für Verkehrssicherheit 03/2010, 228-238.

Mutzenich, C., Durant, S., Helman, S., \& Dalton, P. (2021). Updating our understanding of situation awareness in relation to remote operators of autonomous vehicles. Cognitive Research: Principles and Implications, 6(1), 9. https://doi.org/10.1186/s41235-021-00271-8

Neumeier, S., Wintersberger, P., Frison, A. K., Becher, A., Facchi, C., \& Riener, A. (2019). Teleoperation: The holy grail to solve problems of automated driving? Sure, but latency matters. In Proceedings of the 11th International Conference on Automotive User Interfaces and Interactive Vehicular Applications (pp. 186-197). https://doi.org/10.1145/3342197.3344534

Pradhan, A. K., \& Crundall, D. (2016). Hazard avoidance in young novice drivers: Definitions and a framework. In D. L. Fisher, J. Caird, W. Horrey, \& L. Trick (Eds.), Handbook of Teen and Novice Drivers - Research, Practice, Policy, and Directions (pp. 61-73). CRC Press.

PrognosAG. (2018). Einführung von Automatisierungsfunktionen in der Pkw-Flotte Auswirkungen auf Bestand und Sicherheit. Forschungsbericht erstellt im Auftrag des ADAC e. V. München. https://www.adac.de//media/pdf/motorwelt/prognos_automatisierungsfunktionen.pdf Zugegriffen: 23. Juli 2021.

Rasmussen, J. (1983). Skills, rules, and knowledge; signals, signs, and symbols, and other distinctions in human performance models. IEEE Transactions on Systems, Man, and Cybernetics, (3), 257-266. 
Reinberg, A., \& Ashkenazi, I. (2008). Internal desynchronization of circadian rhythms and tolerance to shift work. Chronobiology International, 25(4), 625-643.

Richter, P. (2015). Arbeit zwischen Fremd- und Eigenkontrolle - Möglichkeiten und Grenzen humaner Arbeitsgestaltung in der DDR. Psychologie des Alltagshandelns, $8(2), 4-11$.

Scholtz, J., Antonishek, B., \& Young, J. (2004). Operator interventions in autonomous offroad driving: Effects of terrain. In IEEE International Conference on Systems, Man and Cybernetics (IEEE Cat. No.04CH37583), (2797-2802),

Shultz, K. S., Wang, M., \& Olson, D. A. (2010). Role overload and underload in relation to occupational stress and health. Stress and Health, 26(2), 99-111. https://doi.org/10.1002/smi.1268

Society of Automotive Engineers (SAE) (2021) SAE International Standard J3016:

Taxonomy and Definitions for Terms Related to Driving Automation Systems for OnRoad Motor Vehicles. https://saemobilus.sae.org/content/J3016_202104/. Zugegriffen: 25. Juni 2021

Staubach, M. (2009). Factors correlated with traffic accidents as a basis for evaluating Advanced Driver Assistance Systems. Accident Analysis \& Prevention, 41(5), 10251033. https://doi.org/10.1016/j.aap.2009.06.014

UNECE. (2020). Human factors challenges of remote support and control: A position paper from HF-IRADS. Informal Document No. 8-September 2020 session of WP.1, 1-9. https://unece.org/fileadmin/DAM/trans/doc/2020/wp29grva/GRVA-07-65e.pdf Zugegriffen: 23. Juli 2021.

Wagner, T., Strohbeck-Kühner, P., \& Koehl, F. (2018). Straftaten und Verstöße gegen verkehrsrechtliche Vorschriften - Kommentar zu den Begutachtungsleitlinien zur Kraftfahreignung, Kapitel 3.16 und 3.17. In W. Schubert, M. Huetten, C. Reimann, M. Graw, W. Schneider, \& E. Stephan (Eds.), Begutachtungsleitlinien zur Kraftfahreignung - Kommentar (3rd ed., pp. 341-376). Kirschbaum Verlag.

Weber, J. (2020). Bewegende Zeiten: Mobilität der Zukunft. Springer.

Yanco, H. A., \& Drury, J. (2004). "Where am I?" Acquiring situation awareness using a remote robot platform. 2004 IEEE International Conference on Systems, Man and Cybernetics (IEEE Cat. No.04CH37583),

Young, M. S., \& Stanton, N. A. (2002). Attention and automation: New perspectives on mental underload and performance. Theoretical Issues in Ergonomics Science, 3(2), 178-194. https://doi.org/10.1080/14639220210123789 